\title{
Reduction of the Body Weight-adapted Volume of Contrast Material by Increasing the Injection Rate in 320-detector Row Coronary CT Angiography
}

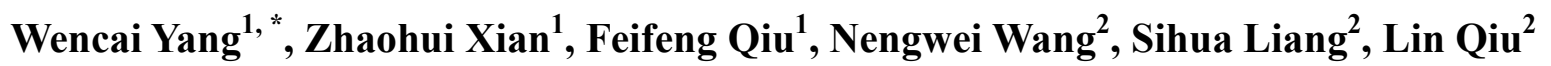 \\ ${ }^{1}$ Interventional Operating Room, The First Affiliated Hospital of Jinan University, Guangzhou, China \\ ${ }^{2}$ Medical Imaging Department, The First Affiliated Hospital of Jinan University, Guangzhou, China
}

Email address:

jnuyang@163.com (Wencai Yang)

*Corresponding author

\section{To cite this article:}

Wencai Yang, Zhaohui Xian, Feifeng Qiu, Nengwei Wang, Sihua Liang, Lin Qiu. Reduction of the Body weight-adapted Volume of Contrast Material by Increasing the Injection Rate in 320-detector Row Coronary CT Angiography. International Journal of Medical Imaging.

Vol. 8, No. 4, 2020, pp. 65-71. doi: 10.11648/j.ijmi.20200804.11

Received: August 24, 2020; Accepted: October 6, 2020; Published: October 16, 2020

\begin{abstract}
Background: 320-detector row dynamic volume computed tomography (CT) scanner was widely applied in coronary CT angiography (CCTA), which made it possible to reduce the volume of contrast material (CM) used. Some studies have reported the feasibility of reducing the CM in 320-detector row CCTA using a weight-adapted injection protocol. However, it hasn't been studied to investigate what was the significance of increasing the injection rate with a lower volume of $\mathrm{CM}$. Objective: To investigate the feasibility of reducing the body weight-adapted volume of $\mathrm{CM}$ by increasing the injection rate in 320-detector row CCTA. Methods: A total of 116 patients who underwent 320-detector row CCTA were divided into three groups. Group A received $0.7 \mathrm{ml} / \mathrm{kg}$ of CM $(350 \mathrm{mg} \mathrm{I} / \mathrm{ml})$ at an injection rate of $5.0 \mathrm{ml} / \mathrm{s}(n=40)$; group B received $0.6 \mathrm{ml} / \mathrm{kg}$ of $\mathrm{CM}$ at $5.5 \mathrm{ml} / \mathrm{s}(n=39)$; group C received $0.5 \mathrm{ml} / \mathrm{kg}$ of CM at $6.0 \mathrm{ml} / \mathrm{s}(n=37)$. A $30-\mathrm{ml} 0.9 \%$ saline chaser was administered after the CM. Enhancement values of the cardiovascular territories and coronary arteries were measured and compared. Image quality was also evaluated and compared among the three groups. Result: Enhancement values of the proximal coronary arterial segments for group $\mathrm{C}$ were significantly lower than those for groups $\mathrm{A}$ and $\mathrm{B}$ (all, $P<0.05$ ), whereas there were no significant differences between groups A and B (all, $P>0.05$ ). Similar statistical results were found in the proportion of proximal coronary arterial segments with enhancement values $\geq 300 \mathrm{HU}$, image quality ratings and the proportion of the main of coronary arterial segments with image quality scores $\geq 3$ on both per-vessel and per-patient analyses. Conclusion: At least $0.6 \mathrm{ml} / \mathrm{kg}$ with $350 \mathrm{mg}$ $\mathrm{I} / \mathrm{ml}$ of $\mathrm{CM}$ at $5.5 \mathrm{ml} / \mathrm{s}$ injection rate was required to achieve sufficient and credible evaluation of the coronary artery in 320-detector row CCTA.
\end{abstract}

Keywords: Coronary CT Angiography, 320-detector Row CT, Contrast Material, Injection Rate, Image Quality

\section{Introduction}

With the rapid advancement of multi-detector computed tomography (CT) and improvements in image quality and acquisition speed, coronary CT angiography (CCTA) has become a standard noninvasive imaging modality, with high spatial and temporal resolution, for the diagnosis of coronary artery disease [1,2]. Unfortunately, patients undergoing CCTA are inevitably exposed to iodinated contrast media (CM) [3]. It has been reported that contrast-induced nephropathy (CIN) is an important iatrogenic complication from use of the CM [4], can have a poor prognosis, and may result in additional healthcare costs [5]. A number of studies have focused on how to prevent CIN, and the core recommendation is to use the lowest possible volume of CM because the incidence of CIN is highly associated with the volume of CM used [6]. Therefore, minimizing the total volume of CM used in CCTA is important, especially for patients with significant coronary artery stenosis who may be exposed to more CM during subsequent coronary artery stenting or angioplasty [7].

In recent years, the 320-detector row dynamic volume CT scanner was widely applied in clinical practice. It has a 
z-coverage width of $160-\mathrm{mm}$ and allows acquisition of the entire heart in a single rotation and within a single heartbeat with a minimum temporal resolution of $175 \mathrm{~ms}$ [8]. The non-helical volume scan mode of the entire heart makes it possible to reduce the volume of CM used [9, 10]. Some studies have reported the feasibility of reducing the $\mathrm{CM}$ volume in 320-detector row CCTA using a weight-adapted injection protocol $[11,12]$. However, to our knowledge few studies have investigated what was the significance of increasing the injection rate with a lower volume of $\mathrm{CM}$ in 320-detector row CCTA. Our study was therefore designed to investigate the feasibility of using the lowest possible volume of $\mathrm{CM}$ with a body weight-adapted injection protocol, by increasing the $\mathrm{CM}$ injection rate and precisely determining the $\mathrm{CM}$ bolus arrival time, to achieve sufficient and credible evaluation of the coronary artery with 320-detector row CCTA.

\section{Materials and Methods}

\subsection{Patients}

This study was performed according to the principles of the Declaration of Helsinki and was approved by our institutional review board. Informed consent was obtained from all patients before the CCTA examination. Before this clinical study, a preliminary retrospective investigation was performed to determine the lowest possible $\mathrm{CM}$ volume and injection rate in 320-detector row CCTA.

From February 2019 to September 2019, a total of 116 patients (68 males and 48 females; mean age, $60.42 \pm 12.35$ years; range, 29 - 86 years) who were scheduled to undergo 320-detector row CCTA were consecutively recruited for this study. All patients were suspected of having coronary artery disease based on electrocardiography (ECG) findings or clinical symptoms, and had no history of coronary artery stenting or bypass surgery. Patients who had a previous allergic reaction to iodinated $\mathrm{CM}$, respiratory failure, severe arrhythmias, congestive heart failure, renal failure (serum creatinine $>1.5 \mathrm{mg} / \mathrm{dl}[133 \mathrm{~mol} / \mathrm{l}]$ ), or an inability to achieve a heart rate below 75 beats per minute (bpm) with the use of beta-blocking agents, as well as women who were potentially pregnant, were excluded. Metoprolol (Metoprolol tartrate tablets, AstraZeneca AB, Sweden) $25 \mathrm{mg}$ or $50 \mathrm{mg}$ as a single dose was administered orally to 23 patients $1-2$ hours before examination in order to meet the heart rate inclusion criterion.

The 116 enrolled patients were randomly divided into three groups using a table of random numbers. Group A $(n=40)$ received $0.7 \mathrm{ml} / \mathrm{kg}$ of $\mathrm{CM}$ at an injection rate of $5.0 \mathrm{ml} / \mathrm{s}$, group $\mathrm{B}(n=39)$ received $0.6 \mathrm{ml} / \mathrm{kg}$ of $\mathrm{CM}$ at $5.5 \mathrm{ml} / \mathrm{s}$, and group $\mathrm{C}(n=37)$ received $0.5 \mathrm{ml} / \mathrm{kg}$ of $\mathrm{CM}$ at $6.0 \mathrm{ml} / \mathrm{s}$. All patients received nonionic CM (Optiray, Ioversol Injection; $350 \mathrm{mg}$ of iodine per milliliter; Tyco Healthcare, Quebec, Canada). A 30-ml 0.9\% saline chaser was administered with the same injection rate after the injection of CM. They were injected using a dual shot injector (Dual Shot Alpha; Nemoto-Kyorindo, Tokyo, Japan) through a 20-gauge or 18-gauge (injection rate of $6.0 \mathrm{ml} / \mathrm{s}$ ) intravenous injection catheter (BD Intima II; Becton Dickinson Medical Devices, New Jersey, USA) inserted into an antecubital vein.

\subsection{CT Scanning}

CCTA was performed using a 320-detector row dynamic volume CT scanner (Aquilion ONE; Toshiba Corporation Medical Systems, Tokyo, Japan). Nitroglycerin spray (0.5 mg, glycerol trinitrate; Jing-wei Pharmaceutical Corp. Ltd. Shandong, China) was administered sublingually 3 - 5 minutes prior to the examination. Prospective ECG gating (Cardiac Trigger Monitor 3000; IVY Biomedical Systems Inc., Branford, USA) was used. The CCTA scanning parameters were: $320 \times 0.5-\mathrm{mm}$ collimation, 0.35 -s gantry rotation time, $120-\mathrm{kV}$ tube voltage, and 350 - $550 \mathrm{~mA}$ tube current.

The delay between the start of the CM injection and scanning was set with the help of automatic bolus-tracking technology (Real Prep technique; Toshiba Corporation Medical Systems, Tokyo, Japan). Dynamic monitoring scanning $(120 \mathrm{kV}$, a tube current of $50 \mathrm{~mA})$ was performed at the midlevel of the heart. Two regions of interest (ROIs) were placed in the left ventricle (LV) and the descending aorta (DA), and the threshold values were set at 100 and 280 Hounsfield units (HU), respectively. Twelve seconds after the initiation of intravenous CM injection, dynamic monitoring scanning was started to obtain the dynamic monitoring image of every second. The patient was instructed to take a breath and hold it when the enhancement value in the LV reached $100 \mathrm{HU}$ (first threshold). After approximately $5.5 \mathrm{~s}$, when the enhancement value in the DA reached $280 \mathrm{HU}$ (second threshold), diagnostic scanning was performed automatically when the patient held his/her breath (Figure 1).

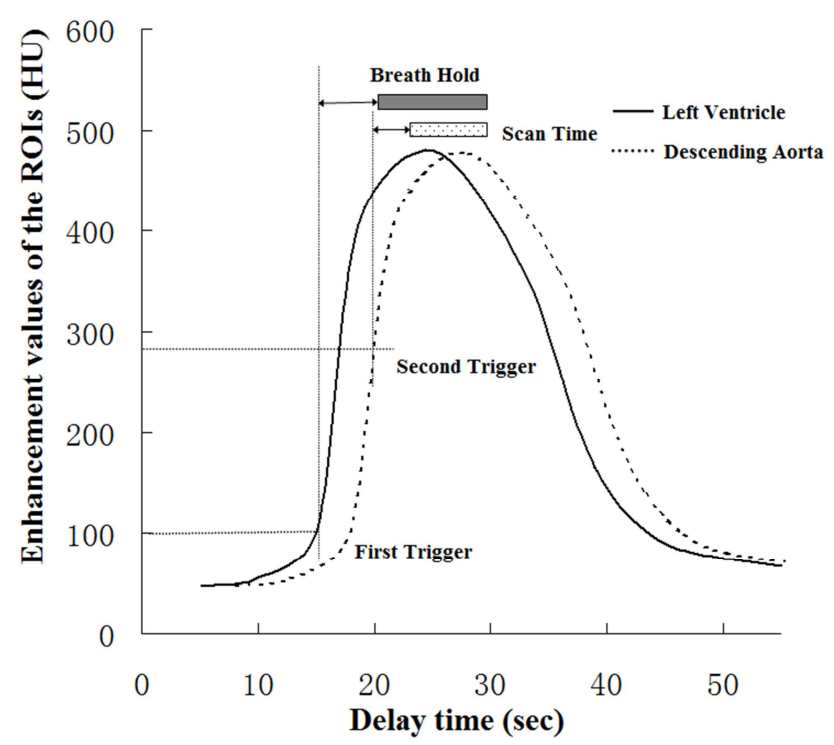

Figure 1. Two regions of interest (ROIs) were placed in the left ventricle $(L V)$ (real line) and the descending aorta (DA) (dashed line).

Reconstruction phase was determined at the system's console by using cardiac-phase search software (Phase Navi; Toshiba Corporation Medical Systems, Tokyo, Japan). Images were reconstructed by using a segmented reconstruction 
algorithm at $75 \%$ of the R-R interval or auto-choose best phase with a slice thickness of $0.5 \mathrm{~mm}$ and a reconstruction interval of $0.25 \mathrm{~mm}$ (image matrix $512 \times 512$ ). If motion artifacts were still present in any coronary artery at this phase, additional reconstructions were performed with the reconstruction window offset by $5 \%$ toward the beginning or end of the cardiac cycle, or at intervals of $10 \mathrm{~ms}$. The image with the fewest motion artifacts among all of the reconstructed images was chosen to transfer to an off-line 3D workstation (Vitrea FX; Vital Images, Minnesota, USA) for post-processing.

\subsection{Data Measurement and Image Quality Evaluation}

Enhancement values of vascular structures were measured in all patients by an experienced radiologist using a manually defined circular ROI cursor. The radiologist was blinded to the injection protocol performed and to the patient grouping. Enhancement values of the cardiovascular territories were measured on axial images at two representative slice levels: level 1 at the origin of the left main coronary artery (LMCA) was used to measure the enhancement values of the ascending aorta (AA) and pulmonary trunk (PT) (Figure 2a), and level 2 at the midlevel of the heart was used to measure the enhancement values of the right atrium (RA), right ventricle (RV), left atrium (LA), LV, and DA (Figure 2b). The mean enhancement values based on two measurements by the radiologist were used for analysis. Enhancement values of the coronary arteries were measured at the following five points on cross-sectional images in which the vessel lumen was easily identified: LMCA, proximal segments of the left anterior descending artery (LAD), left circumflex artery (LCX), right coronary artery (RCA), and distal segments of the RCA. The mean enhancement values based on three measurements obtained from three ROIs in each target point were used for analysis. Figure $2 \mathrm{c}$ depicted the methods of measurement for the point of the proximal RCA (RCA-p). Calcifications, soft plaques, papillary muscles, and areas of stenosis were carefully avoided. To avoid the influence of partial volume effects, the coronary arteries were required to be more than $2 \mathrm{~mm}$ in diameter.
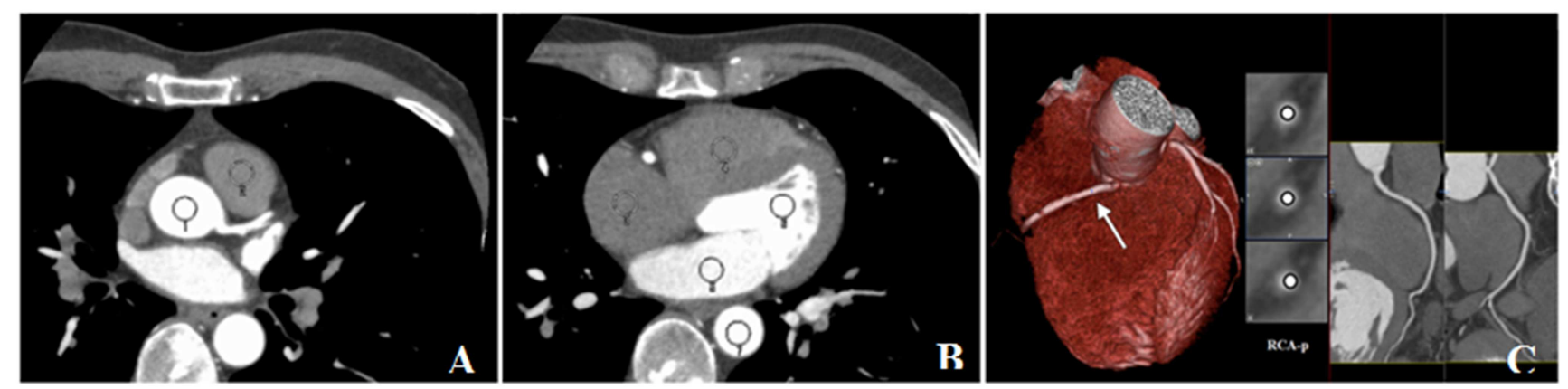

Figure 2. Enhancement values of the cardiovascular territories were measured at the following two representative slice levels: A: level 1 at the origin of the left main coronary artery for the ascending aorta (ROI 1) and pulmonary trunk (ROI 2); B: level 2 at the midlevel of the heart for the right atrium (ROI 3), right ventricle (ROI 4), left atrium (ROI 5), left ventricle (ROI 6), and descending aorta (ROI 7); $C$ : the point of the proximal right coronary artery (RCA-p).

Image quality was evaluated independently by another two cardiovascular radiologists with more than 5 years of experience in cardiac CT imaging using a 5-point grading scale for the main coronary arterial segments: $5=$ excellent, 4=good, 3=acceptable, $2=$ suboptimal, and 1=nondiagnostic. The main segments of the coronary arteries were assessed qualitatively by scoring of all vessels with a diameter of at least a $1.5 \mathrm{~mm}$, including the LMCA and proximal, middle, and distal segments of the LAD, LCX, and RCA. Every segment of the coronary artery with a score of 3 or higher was considered a diagnosable image.

\subsection{Statistical Analysis}

Statistical analysis was performed using SPSS software version 21.0 (SPSS, Chicago, IL, USA). The quantitative data were expressed as the mean \pm standard deviation (SD). The means were compared with one-way analysis of variance (ANOVA) among the three groups. To evaluate the homogeneity of contrast enhancement in the entire coronary artery, a paired $t$-test was used to compare the enhancement value between the proximal and distal portions of the RCA in each group. Statistical significance was accepted at a value of
$P<0.05$. Image quality ratings of the main of coronary arterial segments for the three groups were compared using the nonparametric Kruskal-Wallis test, and pair-wise comparisons of groups were performed using the Mann-Whitney $U$ test. Inter-observer agreements of the image quality ratings were calculated using kappa statistics. Pearson Chi-Square test was performed to examine the difference in the proportions of the proximal coronary arterial segments with enhancement values $\geq 300 \mathrm{HU}$ and the proportion of the main of coronary arterial segments with a score of 3 or higher on per-vessel and per-patient analyses. On the per-patient analysis, the proportion of patients with all proximal segments $\geq 300 \mathrm{HU}$ and all the assessed coronary arterial segments with a score $\geq$ 3 were calculated. In pair-wise comparisons, statistical significance was accepted at a value of $P<0.05 / 3=0.017$ by Bonferroni correction.

\section{Results}

CCTA was performed successfully in all 116 patients without any technical problems or adverse reactions to the CM. Significant coronary artery stenosis (lumen obstruction of $\geq$ 
$50 \%$ ) were noted in 34 patients $(29.31 \%)$. There were no significant differences in demographic characteristics or CT scanning parameters including scan delay, scan time, breath-holding time (BHT), and dose-length product (DLP) among the three groups (all, $P>0.05$ ) (Table 1). The mean total volume of CM used was $46.53 \pm 6.64 \mathrm{ml}$ in group A, $40.59 \pm 6.12 \mathrm{ml}$ in group $\mathrm{B}$, and $33.92 \pm 4.47 \mathrm{ml}$ in group $\mathrm{C}$. The injection duration was $9.70 \pm 1.44 \mathrm{~s}$ in group $\mathrm{A}, 7.85 \pm$ $1.18 \mathrm{~s}$ in group $\mathrm{B}$, and $6.14 \pm 0.75 \mathrm{~s}$ in group $\mathrm{C}$.

Table 1. Patients' characteristics and treatment method of CT scanning for the three groups.

\begin{tabular}{|c|c|c|c|c|c|}
\hline & Group A & Group B & Group C & Statistic & $\boldsymbol{P}$ \\
\hline No. of patients & 40 & 39 & 37 & -- & -- \\
\hline Male/female ratio & $23 / 17$ & $23 / 16$ & $22 / 15$ & $0.033^{*}$ & 0.983 \\
\hline Age (years) & $63.20 \pm 11.01$ & $59.15 \pm 13.63$ & $58.76 \pm 12.12$ & 1.568 & 0.213 \\
\hline Weight $(\mathrm{kg})$ & $65.82 \pm 9.53$ & $67.18 \pm 10.37$ & $67.46 \pm 8.87$ & 0.322 & 0.725 \\
\hline Height (cm) & $164.35 \pm 6.76$ & $162.56 \pm 7.35$ & $163.27 \pm 9.45$ & 0.513 & 0.600 \\
\hline $\mathrm{BMI}\left(\mathrm{kg} / \mathrm{m}^{2}\right)$ & $24.31 \pm 2.72$ & $25.33 \pm 2.97$ & $25.38 \pm 3.31$ & 1.596 & 0.207 \\
\hline Heart rate $(\mathrm{bpm})$ & $61.92 \pm 5.78$ & $60.97 \pm 5.83$ & $61.51 \pm 6.16$ & 0.256 & 0.775 \\
\hline Volume of CM (ml) & $46.53 \pm 6.64$ & $40.59 \pm 6.12$ & $33.92 \pm 4.47$ & 44.620 & 0.000 \\
\hline Injection rate $(\mathrm{ml} / \mathrm{s})$ & 5.0 & 5.5 & 6.0 & - & - \\
\hline Injection duration (s) & $9.70 \pm 1.44$ & $7.85 \pm 1.18$ & $6.14 \pm 0.75$ & 89.920 & 0.000 \\
\hline Scan delay time (s) & $19.86 \pm 2.79$ & $19.88 \pm 2.26$ & $20.11 \pm 3.01$ & 0.100 & 0.905 \\
\hline Scan time (s) & $3.02 \pm 0.42$ & $3.03 \pm 0.41$ & $3.07 \pm 0.48$ & 0.140 & 0.870 \\
\hline BHT (s) & $8.22 \pm 0.69$ & $8.23 \pm 0.73$ & $8.27 \pm 0.80$ & 0.055 & 0.947 \\
\hline $\mathrm{DLP}(\mathrm{mGy} \cdot \mathrm{cm})$ & $245.47 \pm 58.93$ & $248.47 \pm 57.45$ & $258.31 \pm 57.42$ & 0.510 & 0.602 \\
\hline
\end{tabular}

Unless otherwise specified, the data are the means $\pm \mathrm{SD}$; Statistic of the male/female ratio is $\chi^{2}$ value.

$B M I$ Body mass index, $b p m$ beats per minute, $C M$ contrast material, $B H T$ breath-holding time, $D L P$ dose-length product, $S D$ standard deviation.

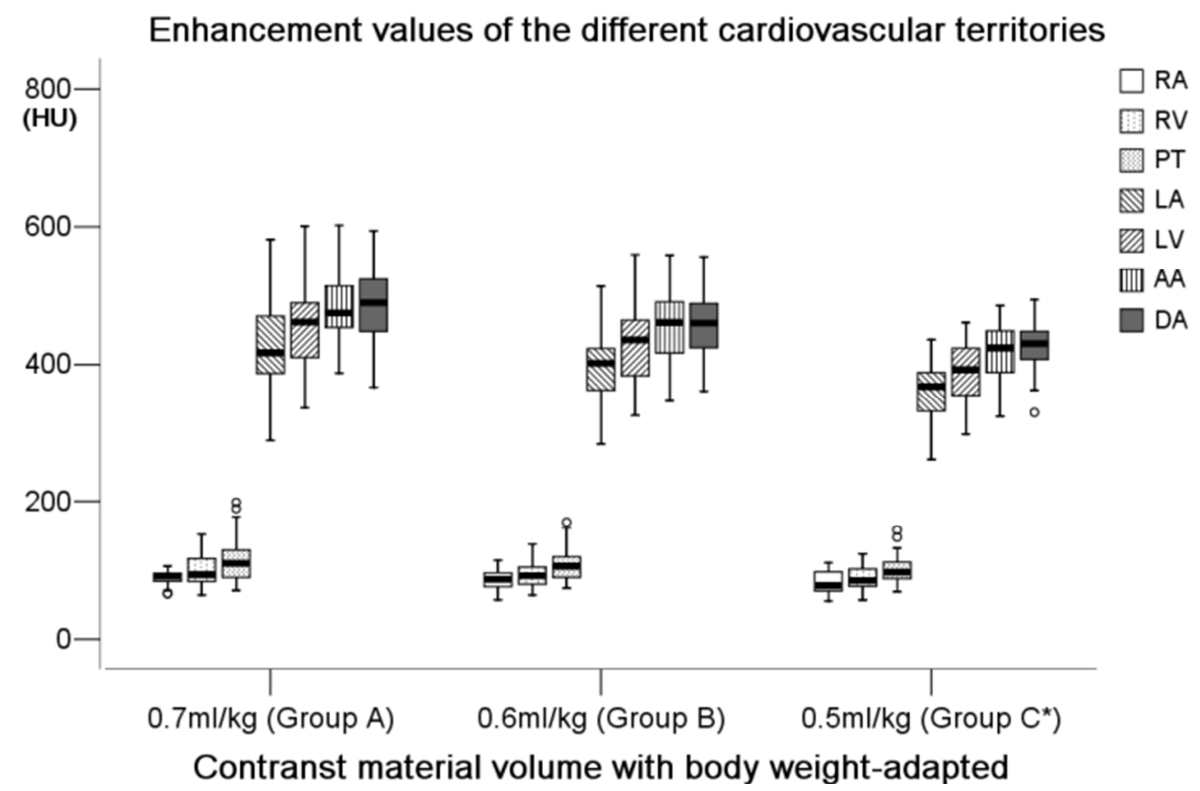

Figure 3. Box plot illustrated enhancement values within the different cardiovascular territories. $* P<0.05$ for group $C$ vs. group A and group $B$ with the mean enhancement values of the $L A, L V, A A$ and $D A$.

Enhancement values of the cardiovascular territories are shown in Figure 3. There were no significant differences in the mean enhancement values of the RA, RV, and PT among the three groups (all, $P>0.05$ ). The mean enhancement values of the LA, LV, AA, and DA exhibited a declining trend from group $\mathrm{A}$ to group $\mathrm{B}$ to group $\mathrm{C}$, which for group $\mathrm{C}$ were lower than those for groups $\mathrm{A}$ and $\mathrm{B}$ (all, $P<0.05)$, whereas there were no significant differences between group A and group B (all, $P>0.05$ ).

The mean enhancement values of the LMCA, proximal LAD (LAD-p), proximal LCX (LCX-p), proximal RCA (RCA-p), and distal RCA (RCA-d) for group $\mathrm{C}$ were lower than those for groups $\mathrm{A}$ and $\mathrm{B}$ (all, $P<0.05$ ), whereas there were no significant differences between group A and group B (all, $P>$ 0.05) (Table 2). There were no significant differences in the enhancement values between the RCA-p and RCA-d in groups $\mathrm{A}$ and $\mathrm{B}(P=0.319, P=0.941$, respectively $)$, whereas a significant difference was noted in group $\mathrm{C}(t=3.269, P=$ $0.002)$. The proportions of the proximal coronary arterial segments with enhancement values $\geq 300 \mathrm{HU}$ on per-vessel and per-patient analysis were $71.17 \%(79 / 111)$ and $62.16 \%(23 / 37)$ in group $\mathrm{C}$ as compared to $90.83 \%(109 / 120)$ and $90.00 \%$ 
(36/40) in group A, and 88.89\% (104/117) and 87.18\% (34/39) in group $\mathrm{B}$, respectively. Those proportions in group $\mathrm{C}$ were significantly lower than those in groups $\mathrm{A}$ and $\mathrm{B}$ on both per-vessel and per-patient analysis (all, $P<0.012$ ), but there were no significant differences between group A and group $\mathrm{B}$ $\left(\chi^{2}=0.248, P=0.620 ; \chi^{2}=0.156, P=0.693\right)$.

Table 2. The mean enhancement values of the coronary arteries for the three groups.

\begin{tabular}{llllll}
\hline & Group A & Group B & Group C & F & 13.725 \\
\hline LMCA & $405.58 \pm 51.79$ & $382.55 \pm 43.74$ & $351.86 \pm 37.94$ & $320.63 \pm 40.31$ & 15.035 \\
LAD-p & $376.89 \pm 51.53$ & $353.56 \pm 42.26$ & $302.88 \pm 40.98$ & 11.589 \\
LCX-p & $353.74 \pm 52.94$ & $329.68 \pm 43.68$ & $335.11 \pm 39.01$ & 21.077 \\
RCA-p & $400.64 \pm 50.18$ & $376.36 \pm 43.48$ & $369.35 \pm 50.51$ & $0.000^{*}$ & $0.000^{*}$ \\
RCA-d & $399.03 \pm 44.85$ & $376.26 \pm 41.57$ & $0.000^{*}$ & 26.813 \\
\hline
\end{tabular}

Data are the means $\pm \mathrm{SD} ; * P<0.05$ for group $\mathrm{C}$ vs. group A and group $\mathrm{B}$

$L M C A$ left main coronary artery, $L A D-p$ proximal left anterior descending artery, $L C X$ - $p$ proximal left circumflex artery, $R C A-p$ proximal right coronary artery, $R C A-d$ distal right coronary artery

Image quality ratings of the main coronary arterial segments were $4.60 \pm 0.83$ or $4.52 \pm 0.92$ in group A, $4.54 \pm$ 0.95 or $4.44 \pm 1.04$ in group $\mathrm{B}$, and $3.87 \pm 1.39$ or $3.72 \pm 1.41$ in group $\mathrm{C}$ by reader 1 or reader 2 , respectively. The image quality for group $\mathrm{C}$ was significantly worse than that for groups A and B (all, $P<0.000$ ), but there were no significant differences between group A and group B $(Z=0.467, P=$ 0.641 or $Z=0.509, P=0.611$ ) with reader 1 or reader 2 . Inter-observer agreements regarding image quality ratings of each measured segment of the coronary arteries were all good $(\kappa \geq 0.730)$. The results of the proportion of the main of coronary arterial segments with a score of 3 or higher on per-vessel and per-patient analysis by reader 1 or reader 2 are shown in Table 3 . The proportions of coronary arteries with a score $\geq 3$ on per-vessel and per-patient analysis for group $\mathrm{C}$ were significantly lower than those for groups A and B (all, $P$ $<0.012)$, but there were no statistically significant differences between group A and group B $\left(\chi^{2}=2.907, P=0.088 ; \chi^{2}=\right.$ 2.414, $P=0.120$ or $\chi^{2}=0.614, P=0.433 ; \chi^{2}=0.156, P=$ $0.693)$.

Table 3. Proportion of the main coronary arterial segments with image quality scores $\geq 3$ for the three groups.

\begin{tabular}{llllll}
\hline & LMCA & LAD (p, m, d) & LCX (p, m, d) & RCA (p, m, d) & Per-vessel analysis \\
\hline Group A & & & & & Per-patient analysis \\
Reader 1 & $40 / 40$ & $114 / 120$ & $111 / 120$ & $120 / 120$ & $385 / 400(96.25 \%)$ \\
Reader 2 & $40 / 40$ & $112 / 120$ & $108 / 120$ & $120 / 120$ & $380 / 400(95.00 \%)$ \\
Kappa & 0.895 & 0.798 & 0.730 & 0.877 & $36 / 40(92.50 \%)$ \\
Group B & & & & & \\
Reader 1 & $38 / 39$ & $111 / 117$ & $102 / 117$ & $114 / 117$ & $365 / 390(93.59 \%)$ \\
Reader 2 & $38 / 39$ & $108 / 117$ & $102 / 117$ & $112 / 117$ & $360 / 390(92.31 \%)$ \\
Kappa & 0.799 & 0.778 & 0.768 & 0.797 & $34 / 39(87.18 \%)$ \\
Group C & & & & & \\
Reader 1 & $34 / 37$ & $78 / 111$ & $71 / 111$ & $96 / 39(87.18 \%)$ \\
Reader 2 & $32 / 37$ & $82 / 111$ & $69 / 111$ & $279 / 370(75.41 \%)^{*}$ \\
Kappa & 0.802 & 0.775 & 0.762 & $94 / 111$ & $277 / 370(74.86 \%)^{*}$ \\
\hline
\end{tabular}

$* P<0.012$ for group $\mathrm{C}$ vs. group $\mathrm{A}$ and group $\mathrm{B}$ on per-vessel and per-patient analysis

$L M C A$ Left main coronary artery, $L A D$ left anterior descending artery, $L C X$ left circumflex artery, $R C A$ right coronary artery, $p$ proximal segment, $m$ middle segment, $d$ distal segment.

\section{Discussion}

In 320-detector row CCTA, volume data acquisition is completed at once by the CT scanner when the coronary artery enhancement arrive peak, and then reconstructs three-dimensional images of the coronary artery with computer post-processing [13]. In general, consistent and high enough vascular contrast enhancement is considered a prerequisite to sufficiently evaluate the coronary artery. And most studies tend to believe this enhancement value shall not be less than $300 \mathrm{HU}$ [14]. Enhancement values of the coronary arteries are suffered from numerous interacting factors, including scan delay, scan time, scan direction and scan mode; CM concentration, volume, injection rate, injection duration, injection mode; patient age, body weight, cardiac output and other factors [15]. Some researchers think body weight is a cardinal patient-related factor affecting the magnitude of vascular contrast enhancement [11, 16], and suggest that the $\mathrm{CM}$ volume is adjusted according to patient body weight in CCTA. On the other hand, injection rate is another primary $\mathrm{CM}$ injecting factor affecting the magnitude of vascular contrast enhancement.

In the current study, we compared the three groups according to the volume of $\mathrm{CM}$ administered with a body weight-adapted injection protocol: patients received $0.7 \mathrm{ml} / \mathrm{kg}$ of CM at an injection rate of $5.0 \mathrm{ml} / \mathrm{s}$ (group A), $0.6 \mathrm{ml} / \mathrm{kg}$ of $\mathrm{CM}$ at $5.5 \mathrm{ml} / \mathrm{s}$ (group B), or $0.5 \mathrm{ml} / \mathrm{kg}$ of $\mathrm{CM}$ at $6.0 \mathrm{ml} / \mathrm{s}$ (group $\mathrm{C}$ ). The results showed there were no significant differences in the mean enhancement values of the coronary artery and the image quality between group A and group B. This demonstrates that increasing the $\mathrm{CM}$ injection rate allows a $12.77 \%$ reduction in the total volume of $\mathrm{CM}$ from $0.7 \mathrm{ml} / \mathrm{kg}$ (mean $46.53 \mathrm{ml}$ ) to $0.6 \mathrm{ml} / \mathrm{kg}$ (mean $40.59 \mathrm{ml}$ ) for CCTA 
without affecting the imaging quality. We tried to reduce the volume of $\mathrm{CM}$ to $0.5 \mathrm{ml} / \mathrm{kg}$ (mean $33.92 \mathrm{ml}$ ). But the mean enhancement values in the cardiovascular territories and coronary arteries all decreased significantly, and the image quality was also destroyed.

Using a lower $\mathrm{CM}$ volume and higher injection rate implies that the injection duration and peak time of contrast enhancement in the coronary artery will be shorter [13]. If the injection duration is too short, the bolus will be not enough to maintain adequate enhancement during the volume data acquisition, which will lead to inhomogeneous enhancements between the proximal and distal portions of the coronary artery. In our study, the injection duration was $9.70 \pm 1.44 \mathrm{~s}$ in group A, $7.85 \pm 1.18 \mathrm{~s}$ in group B, and $6.14 \pm$ $0.75 \mathrm{~s}$ in group $\mathrm{C}$. And the results showed no significant difference in the enhancement values between the proximal and distal portions of RCA in groups $\mathrm{A}$ and $\mathrm{B}$, indicating that the injection duration was adequate. However, the enhancement values of the proximal and distal portions of the RCA were significantly different in group $\mathrm{C}$, demonstrating it was difficult to maintain consistent contrast enhancement in the whole coronary artery. In consideration of partial volume effects, measurements were not taken in the distal portions of LAD and LCX because the diameter of these vessels was $\leq 1.5 \mathrm{~mm}$ in most patients.

With a lower CM volume and shorter injection duration, exact determination of $\mathrm{CM}$ arrival time is crucial to synchronize CT image acquisition for optimal coronary enhancement Determination of CM arrival time is typically done using either the test bolus (TB) technique or automatic bolus-tracking (BT) technique. However, the TB has been abandoned because it requires the use of a small volume (10 $20 \mathrm{ml}$ ) of CM, which is incompatible with the goal of reducing the CM volume used in CCTA. The automatic BT is based on real-time monitoring of the main bolus during injection with the acquisition of a series of dynamic low-dose monitoring scans at the midlevel of heart, where the CM can be visually observed passing through the RA, RV, and pulmonary circulation and finally reaching the LA and LV at the "first pass". Tatsugami et al. reported that the use of a two-threshold setting in the AA could reduce inter-patient variability using the automatic trigger mode of BT in 320-detector row CTCA [17]. Nevertheless, when the two thresholds are set in the AA, the patient only has approximately $3 \mathrm{~s}$ to complete the movement of taking a breath and holding it, which is difficult to achieve, especially for some elderly patients. In our study, we selected two ROIs in the LV and the DA with threshold values of 100 and $280 \mathrm{HU}$, respectively. When the $\mathrm{CM}$ arrived at the LA, the first threshold $(100 \mathrm{HU})$ was triggered to instruct the patient to take a breath and hold it. After approximately $5.5 \mathrm{~s}$, the enhancement value in the DA reached $280 \mathrm{HU}$ (second threshold); the diagnostic scan was performed automatically while the patient was holding his/her breath. The mean BHT was $8.22 \pm 0.69 \mathrm{~s}$ in group A, $8.23 \pm$ $0.73 \mathrm{~s}$ in group $\mathrm{B}$, and $8.27 \pm 0.80 \mathrm{~s}$ in group $\mathrm{C}$. Compared with the traditional methods in which the patient is instructed to begin the breath hold at $14 \mathrm{~s}$ after the initiation of intravenous
CM injection, this method shortens the BHT, especially for some patients with reduced pulmonary circulation. It also avoids the respiratory motion artifacts.

\section{Conclusion}

In conclusion, a total of at least $0.6 \mathrm{ml} / \mathrm{kg}$ with a $350 \mathrm{mg} \mathrm{I} / \mathrm{ml}$ concentration of $\mathrm{CM}$ at $5.5 \mathrm{ml} / \mathrm{s}$ injection rate are required to achieve sufficient and credible evaluation of the coronary artery in 320-detector row CCTA. Increasing the injection rate can compensate for the lower coronary arterial enhancement caused by the minimized $\mathrm{CM}$ volume to some extent.

\section{References}

[1] Sebastian B, Mohamed M, Jörg H, et al. Coronary computed tomography angiography (CCTA) in patients with suspected stable coronary artery disease (CAD): diagnostic impact and clinical consequences in the German Cardiac CT Registry depending on stress test results [J]. The International Journal of Cardiovascular Imaging (formerly Cardiac Imaging), 2019, 35 (4): 741-748.

[2] Fuchs A, Kühl JT, Chen MY, et al. Subtraction CT angiography improves evaluation of significant coronary artery disease in patients with severe calcifications or stents-the C-Sub 320 multicenter trial.[J]. European Radiology. 2018, 28 (10): 4077-4085.

[3] Matthews E. Acute Kidney Injury and Iodinated Contrast Media [J]. Radiologic Technology, 2018, 89 (5): 467CT-477CT.

[4] Jai-Sing Y, Yan-Ru P, Shih-Chang T, et al. The molecular mechanism of contrast-induced nephropathy (CIN) and its link to in vitro studies on iodinated contrast media $(\mathrm{CM})[\mathrm{J}]$. Biomedicine, 2018, 8 (1): 1-11.

[5] Sun G, Chen JY, Liu Y. Contrast-Induced Nephropathy: Further Investigations About Risk Factors Are Required [J]. Angiology. 2019, 70 (8): 784-785.

[6] Hossain M, Costanzo E, Cosentino J, et al. Contrast-Induced nephropathy: Pathophysiology, risk factors, and prevention [J]. Saudi journal of kidney diseases and transplantation: an official publication of the Saudi Center for Organ Transplantation, Saudi Arabia, 2018, 29 (1): 1-9.

[7] Bao L. Optimization of contrast dose of the 320-slice spiral CT coronary angiography [J]. Journal of Imaging Research and Medical Applications, 2018, 2 (13): 37-38.

[8] Ognard J, Dissaux B, Haioun K, et al. A "one-stop-shop" 4D CTA protocol using 320-row CT for advanced imaging in acute ischemic stroke: a technical note [J]. European Radiology, 2019, 29 (9): 4930-4936.

[9] Shirasaka T, Nagao M, Yamasaki Y, et al. Feasible scan timing for 320-row coronary CT angiography generated by the time to peak in the ascending aorta [J]. Clinical imaging, 2019, 54: 153-158.

[10] Al'Aref S J, Min J K. Cardiac CT: current practice and emerging applications [J]. Heart, 2019, 105 (20): 1597-1605.

[11] Qiang L, Li-Na S, Xiang-Wu M A. 320-Slice Volume CT Double Low Technique Applied to the Study of Coronary Artery CTA in Large Body Weight Patients [J]. Chinese Journal of CT and MRI, 2018, 16 (09): 51-53+153. 
[12] Sheng-Jia G U, Le Q, Ze-Peng M A, et al. A Prospective Study of Optimized Contrast Media Injection Protocol for Coronary Computed Tomography Angiography [J]. Chinese Computed Medical Imaging, 2018, 24 (01): 32-37.

[13] Kawaguchi N, Kurata A, Kido T, et al. Optimization of coronary attenuation in coronary computed tomography angiography using diluted contrast material [J]. Circulation journal, 2014, 78 (3): 662-670.

[14] Bae, YK. Intravenous contrast medium administration and scan timing at CT: considerations and approaches [J]. Radiology, 2010, 256 (1): 32-61.

[15] Bae K T, Seeck B A, Hildebolt C F, et al. Contrast enhancement in cardiovascular MDCT: effect of body weight, height, body surface area, body mass index, and obesity [J]. American Journal of Roentgenology, 2008; 190 (3): 777-784.

[16] Sharma R K, Gottlieb I, João A. C. Lima. Cardiovascular CT for Perfusion and Delayed Contrast Enhancement Imaging [M] / Cardiac CT Imaging. Springer International Publishing, 2016: 211-220.

[17] Tatsugami F, Awai K, Takada H, et al. Reduction of interpatient variability of arterial enhancement using a new bolus tracking system in 320-detector computed tomographic coronary angiography [J]. Journal of Computer Assisted Tomography, 2013, 37 (1): 79-83. 